\title{
A review of diffusion tensor imaging studies in schizophrenia
}

\section{Citation}

KUBICKI, M, R MCCARLEY, C WESTIN, H PARK, S MAIER, R KIKINIS, F JOLESZ, and M SHENTON. 2007. A Review of Diffusion Tensor Imaging Studies in Schizophrenia. Journal of Psychiatric Research 41, no. 1-2: 15-30. doi:10.1016/j.jpsychires.2005.05.005.

\section{Published Version}

doi:10.1016/j.jpsychires.2005.05.005

\section{Permanent link}

http://nrs.harvard.edu/urn-3:HUL.InstRepos:33766568

\section{Terms of Use}

This article was downloaded from Harvard University's DASH repository, and is made available under the terms and conditions applicable to Other Posted Material, as set forth at http:// nrs.harvard.edu/urn-3:HUL.InstRepos:dash.current.terms-of-use\#LAA

\section{Share Your Story}

The Harvard community has made this article openly available.

Please share how this access benefits you. Submit a story.

Accessibility 


\title{
A review of diffusion tensor imaging studies in schizophrenia
}

\author{
Marek Kubicki ${ }^{a, b}$, Robert McCarley ${ }^{a,{ }^{*}}$, Carl-Fredrik Westin ${ }^{b}$, Hae-Jeong Park ${ }^{a, b, d}$, Stephan \\ Maier $^{\mathrm{C}}$, Ron Kikinis ${ }^{\mathrm{b}}$, Ferenc A. Jolesz ${ }^{\mathrm{c}}$, and Martha E. Shenton ${ }^{\mathrm{a}, \mathrm{b}},{ }^{*}$ \\ a Clinical Neuroscience Division, Laboratory of Neuroscience, Boston VA Health Care System- \\ Brockton Division, Department of Psychiatry, Harvard Medical School, 940 Belmont Street, \\ Brockton, Boston, MA 02301, United States \\ b Surgical Planning Laboratory, MRI Division, Department of Radiology, Brigham and Women's \\ Hospital, Harvard Medical School, Boston, MA, United States \\ c Department of Radiology, Brigham and Women's Hospital, Harvard Medical School, Boston, MA, \\ United States \\ d Department of Diagnostic radiology, Yonsei University, College of Medicine, Seoul, Republic of \\ Korea
}

\begin{abstract}
Both post-mortem and neuroimaging studies have contributed significantly to what we know about the brain and schizophrenia. MRI studies of volumetric reduction in several brain regions in schizophrenia have confirmed early speculations that the brain is disordered in schizophrenia. There is also a growing body of evidence suggesting that a disturbance in connectivity between different brain regions, rather than abnormalities within the separate regions themselves, are responsible for the clinical symptoms and cognitive dysfunctions observed in this disorder. Thus an interest in white matter fiber tracts, subserving anatomical connections between distant, as well as proximal, brain regions, is emerging. This interest coincides with the recent advent of diffusion tensor imaging (DTI), which makes it possible to evaluate the organization and coherence of white matter fiber tracts. This is an important advance as conventional MRI techniques are insensitive to fiber tract direction and organization, and have not consistently demonstrated white matter abnormalities. DTI may, therefore, provide important new information about neural circuitry, and it is increasingly being used in neuroimaging studies of psychopathological disorders. Of note, in the past five years 18 DTI studies in schizophrenia have been published, most describing white matter abnormalities. Questions still remain, however, regarding what we are measuring that is abnormal in this disease, and how measures obtained using one method correspond to those obtained using other methods? Below we review the basic principles involved in MR-DTI, followed by a review of the different methods used to evaluate diffusion. Finally, we review MR-DTI findings in schizophrenia.
\end{abstract}

\section{Keywords}

Diffusion tensor imaging; Schizophrenia; White matter; Fiber tracts

(C) 2005 Elsevier Ltd. All rights reserved.

* Corresponding authors. Tel.: +1 508583 4500x1371/2473; fax: +1 508580 0059. robert_mccarley@ hms.harvard.edu (R. McCarley), martha_shenton@hms.harvard.edu (M.E. Shenton).. 


\section{Introduction}

While a great deal of progress has been made in delineating gray matter abnormalities in schizophrenia using magnetic resonance imaging (MRI) (for a review see Shenton et al., 2001), far less progress has been made in evaluating white matter abnormalities, or in evaluating white matter fiber tracts interconnecting brain regions, particularly those that connect the frontal and temporal lobes, tracts that have long been thought to be abnormal in schizophrenia (e.g., Wernicke, 1906; Kraepelin, 1919/1971).

Of particular note, unlike studies of gray matter, MR structural studies of white matter have not been as informative. More specifically, only a small number of MRI studies have evaluated white matter volume differences between patients with schizophrenia and controls, and these findings have been largely negative (e.g., Suddath et al., 1990; Wible et al., 1995). Moreover, in one of the few studies reporting white matter volume reduction in schizophrenia, Breier and coworkers (Breier et al., 1992) noted a correlation between prefrontal white matter reduction and amygdala-hippocampal complex volume reduction, thus highlighting the potential importance of frontal-temporal interactions in schizophrenia. More recently, two studies using voxel-based analyses have shown white matter abnormalities in both temporal and frontal lobe regions (Sigmudsson et al., 2001), and bilaterally in the frontal lobe (Paillere-Martinot et al., 2001).

There are also only a few post-mortem studies of white matter in schizophrenia, and these findings, similar to the MRI findings, are inconclusive. Specifically, two recent studies report decreased fiber number and density in the anterior commissure and the corpus callosum in women but not in men with schizophrenia (Highley et al., 1999a,b), and no differences in the number and density of fibers in the uncinate fasciculus (Highley et al., 2002). There is also growing evidence to suggest that glial cells, particularly oligodendrocytes, which form myelin sheaths around axons, are abnormal in schizophrenia (Hakak et al., 2001; Uranova et al., 2001, 2004). For example, Hakak et al. study reported abnormal expression of myelin related genes in schizophrenia, which suggests a disruption in oligodendrocyte function. Furthermore, Uranova et al. (2001) study, using electron microscopy, showed both qualitative and quantitative abnormalities in post-mortem brains of schizophrenics in the oligodentroglia in the prefrontal cortex and caudate nucleus, including a marked increase in the density of concentric lamellar bodies (indicating damage to myelinated fibers) in the caudate nucleus in post-mortem brains of patients diagnosed with schizophrenia, as well as decreased density of the oligodendrocytes in layer IV of the prefrontal cortex in schizophrenia (Uranova et al., 2004). In another study, Hof et al. (2003) found decreased oligodendrocyte number and density in layer III of Brodmann area 9 and in gyral prefrontal white matter in schizophrenia.

In addition, Benes' (1993) work suggests delayed myelination in the prefrontal cortex in patients with schizophrenia. Importantly, if glial dysfunction is confirmed in schizophrenia, this alone could explain abnormal neuronal cytoarchitecture and functional deficits including functional fronto-temporal disconnections observed in schizophrenia (see also the recent review of white matter in schizophrenia by Davis et al. (2003). Finally, Akbarian et al. (1996) reported a maldistribution of the interstitial neurons in both prefrontal and temporal white matter in schizophrenia brains. The observed altered neuronal distributions in both prefrontal and temporal lobe regions suggested to these investigators that neurodevelopmental abnormalities were present in at least a subgroup of patients, and that such abnormalities could alter the connectivity of these brain regions.

Taken together, these studies serve to underscore the importance of evaluating white matter fiber tract abnormalities in schizophrenia. 
Below, we provide a brief review of the principles of DTI, including some of the different measures employed, followed by a review of the findings to date in schizophrenia. For the selection of published DTI studies in schizophrenia, we used PubMed and Medline, where we used the key words diffusion tensor imaging and schizophrenia. We end with a review of future applications of DTI techniques to schizophrenia, which will likely further our understanding of the neuropathology of schizophrenia.

\section{The theory behind diffusion tensor imaging}

One of the main reasons for inconsistent findings regarding white matter abnormalities in schizophrenia is the difficulty in evaluating this brain compartment using conventional MRI. This is primarily because white matter appears homogeneous in conventional MRI, as this methodology is not sensitive to discerning fiber tract direction and/or organization. In contrast, recently, diffusion tensor MRI techniques have been developed which have proven to be useful in the evaluation of different white matter components. DTI is, in fact, an exciting neuroimaging technique that affords a unique opportunity to quantify the diffusion of water in brain tissue. It is based upon the phenomenon of water diffusion known as Brownian motion, named after the English botanist Robert Brown, who in 1827 observed the constant movement of minute particles that were suspended within grains of pollen. As this molecular motion is affected by the properties of the medium in which it occurs, diffusion within biological tissues reflects both tissue structure and architecture at the microscopic level. Further, due to the fact that diffusion is not uniform throughout the brain (differing, for example, between gray matter, white matter, and cerebrospinal fluid), researchers can use this characteristic of DTI to evaluate different brain tissue.

Specifically, in brain tissue, the motion of water molecules can be restricted at many levels, as for example by interacting with different tissue components, such as cell membranes, macromolecules, fibers, myelin sheaths and fiber tracts. Thus within white matter, the mobility of water is restricted in directions that are perpendicular to the fiber tracts. (Parenthetically, this restricted mobility of water is described as anisotropic, whereas unrestricted mobility of water, such as would be observed for cerebrospinal fluid, is described as iso-tropic.) And, while myelination is not essential for diffusion anisotropy of nerves, see for example studies of nonmyelinated garfish olfactory nerves (Beaulieu and Allen, 1994) and studies of neonate brains prior to the appearance of myelin (Huppi et al., 1998), tightly packed multiple myelin membranes accompanying axons are generally assumed to be the major barrier to diffusion in myelinated fiber tracts. Moreover, the tissue properties of white matter fiber tracts, including the density of the fibers, the average fiber diameter, the thickness of the myelin sheaths, and the directionality (or coherence) of the fibers in each voxel, all affect the diffusion of water molecules, and thus, in turn, provide useful information about white matter fiber tract organization. Hence because of the physical properties of white matter, diffusion tensor imaging can be used to investigate white matter in the brain in a manner not possible with conventional MRI imaging techniques.

Since its introduction to clinical use in 1986 by Le Bihan et al. (1986), brain diffusion MRI has become one of the most important tools in clinical neuroimaging. Using this technique, diffusion is encoded in the MRI signal by magnetic field gradient pulses. Accordingly, the displacement of water molecules (diffusion) causes randomization of the NMR spin phase, which, in turn, results in signal reduction. The amount of reduction provides a quantitative measure of the diffusion in the gradient direction, and thus only diffusion in the direction of this particular gradient can be detected. Since diffusion is truly a three-dimensional process, at least three orthogonal measurements are needed to calculate the mean diffusion for each voxel. Basser et al. (1994) introduced a multi-dimensional approach to the assessment of diffusion data in vivo, called diffusion tensor imaging (DTI), followed by the first clinical 
application in 1996 (Pierpaoli et al., 1996). This method requires at least six different, noncollinear and non-planar diffusion measurements, and provides the rotationally invariant (independent to the gradient and head position) estimations of the diffusion and diffusion anisotropy. The diffusion for each voxel is then described as a $3 \times 3$ matrix and called a diffusion tensor. In an isotropic media, such as cerebrospinal fluid, where diffusion in all three main axes is equal, the tensor is symmetrical in all directions, and it can be visualized as a sphere. In an anisotropic media, where the diffusion is different along each axis, the tensor is visualized as an ellipsoid, with its longest axis pointing toward the longest of the so-called principal directions (see Fig. 1).

The shape of the tensor ellipsoid thus depends upon the strength of the diffusion along three principal directions (i.e., its eigenvectors). The geometric nature of the diffusion tensors can then be used to characterize quantitatively the local structure in tissues such as white matter in the brain, because the direction of the major diffusion axis will always be aligned in the direction of the white matter fiber tracts. The DTI images are acquired on high field, high performance MR systems, and analyzed in terms of diffusion anisotropy using dedicated software.

\section{Acquisition of diffusion tensor images in the brain}

In order to detect water diffusion along different directions, Stejskal and Tanner (1965) method is used. This method uses two strong gradient pulses, symmetrically positioned around a refocusing pulse, allowing for diffusion weighting. To eliminate the dependence of spin density, T1 and T2, at least two measurements of diffusion-weighting must be obtained, that are differently sensitized to diffusion but remain identical in all other respects.

As noted previously, in order to correctly assess the diffusion tensor, data from at least six independent diffusion encoding directions is acquired for each voxel. The most popular acquisition technique to obtain DTI images is a single-shot diffusion-weighted, echo-planar (EPI) protocol, introduced in 1990 by Turner et al. (1990). It is fast, thus being robust to motion artifacts, although it is limited in spatial resolution, and very sensitive to image artifacts and distortions.

While DTI EPI remains the most popular technique to acquire diffusion tensor images, other protocols have emerged as alternatives to the low resolution EPI technique. Line-scandiffusion-imaging (LSDI), for example, was introduced by Gudbartsson et al. (1996). Unlike the single-shot EPI, where a complete image is acquired after each radio-frequency excitation, LSDI is based on the sequential acquisition of parallel columns lying in the image plane. The sequential collection of this line data in independent single-shot acquisitions makes the sequence largely insensitive to bulk motion artifacts since no phase encoding is used. Because of the acquisition method, however, the LSDI sequence is four to six times slower than singleshot diffusion-weighted EPI. On the other hand, it allows for better spatial resolution, which is especially important when small fiber bundles are being evaluated. It is also relatively free from the artifacts inherent in single-shot EPI methods. Another advantage of this technique is that it can be used on higher field strengths, with signal to noise $(\mathrm{S} / \mathrm{N})$ gain, and without the dramatic increase of the aforementioned artifacts.

More recently several new techniques have been proposed that further minimize susceptibility and chemical shift artifacts, as well as allowing for higher than EPI resolution and/or signal to noise ratio, and shorter than LSDI scan times. These newer techniques include: (1) diffusion weighted radial acquisition of data (DIFRAD) (Trouard et al., 1999); (2) slab scan diffusion imaging (SSDI) (Maier, 2001); (3) segmented EPI with navigator echoes correction (Ordidge et al., 1994); and (4) single-shot EPI with parallel imaging (Bammer et al., 2002). 
DTI acquisitions are generally performed on high field, high performance gradient systems where 3-4 Tesla magnets are becoming more and more popular in clinical research. However, the 1.5 Tesla magnet is still the preferred MR field strength for clinical imaging. Lower field strengths systems require longer acquisition times (due to the increased number of repetitions to compensate for the decreased signal to noise ratio). Higher field strengths, on the other hand, allow for potentially faster acquisitions and higher $\mathrm{S} / \mathrm{N}$ ratios, but they are not free of limitations. Such limitations, in fact, include (depending on the acquisition technique), eddy current distortions, susceptibility and chemical shift artifacts as well as geometrical distortions of the images, which are, at least in the case of the faster techniques, much higher that on lower field strength machines. Nonetheless, the development of acquisition tools such as high order shimming, which make possible better quality images, as well as post-processing tools, which minimize image distortions and artifacts, should all help to ensure that clinical research can be moved to higher field magnets.

To return, however, to the image acquisition, and as mentioned previously, in order to calculate the tensor, at least six non-colinear measurements of diffusion are required. Some recent schizophrenia studies, however, use even more diffusion directions (Wang et al., 2003; Sun et al., 2003). Of note, multiple direction acquisitions have numerous advantages, including the fact that multiple direction acquisitions allow for the creation of more symmetrical tensors, they minimize noise, as well as make the guiding of fiber tracking more precise. Moreover, higher angular data can be used to solve the problem of fiber crossings, and to guide fiber tracking in areas where the anisotropy is low, and where uncertainty exists with respect to the major direction of diffusion (Tuch et al., 2002; Frank, 2002). This approach, however, requires complicated mathematical models such as in the recent work by Hagmann et al. (2004). In this work, the authors show tractography results of fiber crossing in the centrum semiovale. We note, however, that while the direction of this work is promising, it needs further development and is not validated at this time.

Two final issues that should be taken into account when planning a diffusion experiment, both of which influence the $\mathrm{S} / \mathrm{N}$ ratio, are the number of excitations (NEX), or repetitions, and the image resolution. The $\mathrm{S} / \mathrm{N}$ ratio for single-shot EPI is relatively low (due to movement artifacts and short excitation times), and in order to increase it, signal either needs to be averaged over many repeated scans, or scan resolution needs to be decreased. The first results in longer scanner times, while the second results in less detailed data. Because of motion-related phase variations in the MR signal, complex averaging cannot be applied, but magnitude signal must be used instead. This entails that not only the signal, but also the noise is being averaged. It is advisable, for a good estimation of FA, to maintain an SNR for the diffusion-weighted image data of 10:1. SNR values below 3:1 result in a Rician noise distribution that requires special treatment if magnitude signal averaging is performed. For very low SNR $(<3: 1)$ signal averaging does not eliminate the overestimation of FA values, however, it does improve the estimation of the diffusion tensor eigenvector direction. Of note, in terms of the $\mathrm{S} / \mathrm{N}$ ratios as well as data reproducibility, LSDI performs similar to the single-shot EPI with 16 NEX's (see Kubicki et al., 2004; see also Fig. 2).

The resolution of the images is the other important issue that needs to be considered. Fast MR techniques used for DTI data acquisition do not usually allow for high image resolution, or if they do, the increase in resolution is done at the expense of larger image distortions. Since most white matter structures are relatively small, the partial volume effect often exceeds the size of the fiber bundle. To avoid this problem, one can use large ROIs, and instead of looking at particular fiber tracts focus on the fiber coherence in the larger areas of the white matter. An alternative approach is to use image acquisitions that are perpendicular to the measured fiber (see Fig. 3). Unfortunately, this approach is less efficient, as it can be used only for a very few brain structures (e.g., cingulate bundle, corpus callosum, a portion of the uncinate fasciculus, 
and the internal capsule). For all of the reasons mentioned before, the most optimal method for data acquisition (when permitted by the scanner software and hardware), is to use as small as possible, isotropic voxels. This approach is also preferable if fiber tracking is to be applied to the DTI data.

\section{Quantitative representation of diffusion tensor}

Data from diffusion tensor imaging can be analyzed in several ways. The most general approach is to characterize the overall displacement of the molecules (average ellipsoid size) by calculating the mean diffusivity. To do so, the trace (Tr) (Basser, 1995) of the diffusion tensor, which is calculated as the sum of the eigenvalues of the tensor, has been introduced. The mean diffusivity is then given by $\operatorname{Tr}(D) / 3$.

To describe the anisotropy of diffusion, several scalar indices have been introduced. These indices are rotationally invariant, i.e., not dependent on the MR gradients orientation, or absolute orientation of the diffusion tensor ellipsoid, and thus provide the ultimate value for a specific tissue type. The most commonly used indices, proposed by Basser and colleagues (Basser, 1995) are: (1) relative anisotropy (RA), a normalized standard deviation representing the ratio of the anisotropic part of the tensor to its isotropic part; (2) fractional anisotropy (FA), a measure of the fraction of the magnitude of the tensor that can be ascribed to the anisotropic diffusion; and, (3) volume ratio (VR), a measure representing the ratio of the ellipsoid volume to the volume as a sphere of radius $\lambda$. Other anisotropy indices, such as indices measuring intervoxel coherence (Pfefferbaum et al., 2000), and the geometric nature of diffusion and intravoxel coherence (Westin et al., 2002), have also been proposed, and are reviewed in detail by Kubicki et al. (2002b) (for examples of maps representing diffusion indices see Fig. 3).

Similar to anisotropy indices, several methods have also been proposed for visualizing the 3D information contained in DT-MRI data. These include: (1) the use of the octahedral icon for each pixel (Reese et al., 1995); (2) color maps, where different intensities of the three colors indicate the size and the ADC in each of the three Cartesian directions (Makris et al., 1997); (3) rendered ellipsoids to visualize diffusion data in a slice, and (Pierpaoli et al., 1996); and (4) the use of lines to represent the in-plane component of the principal diffusion direction, along with a color-coded out-of-plane component (Peled et al., 1998) (see Fig. 4).

Finally, multiple methods have been introduced for the quantitative analysis of diffusion. The most popular approach to data analysis in clinical studies is to use a fixed size (square or circle) ROI method. This approach has been used to place large ROIs within anatomically non-specific white matter regions of the brain (i.e., frontal white matter), as well as much smaller ROIs, placed within the specific white matter fiber tracts. These two methods are distinct not only because one is averaging over a bigger area, and thus is less sensitive to the noise and artifacts, and more statistically conservative (Lim et al., 1999), while the other is more anatomically specific. Also, in terms of underlying pathology, anisotropy differences detected by large ROIs, point to the anomalies in coherence and/or organization of the white matter structures within the analyzed region, while differences detected by small ROIs, placed in the middle of the fiber tract, demonstrate abnormalities that could be due to the number/density of the fibers traveled within the fiber tract, as well as their myelin content. While the latter method relates more directly to the question of anatomical connectivity subserved by the measured fiber tract, it still has many limitations. Specifically, small ROI measurements are characterized by larger errors, due to the noise inherent in DTI images. Also, due to the various sizes of the structures across subjects, the sampled region is not always comparable. Lastly, but not the least limitation of the small ROI method, is the partial volume effect. This effect, due to the anisotropic size of voxels used in most of the studies so far, is much higher in the slice direction, than in-plane. What this means is, that in order to minimize the partial volume effect, and thus to get the most 
reliable measurements of the small fiber tract, one should consider (if an acquisition of high resolution images with isotropic voxels is not an option) using an acquisition that is perpendicular to the fiber bundle of interest (see Fig. 5).

An alternative approach for diffusion data analysis is to define the ROI based on the directional information obtained from the DTI. While this method is more specific to the fiber tracts comparing to the small ROI approach (this method extracts cross-section of the whole fiber bundle, as opposed to the fixed square that includes the center of the fiber bundle as in the fixed, small ROI approach), it also has numerous limitations. For example, similar to the small fixed ROI method, the directional threshold should be only applied to the fibers that are perpendicular to the plane, which limits the number of possible bundles tested. Moreover, the approach uses eigenvalues and eigenvectors to define the ROI, and these same variables are then used to calculate the FA within the segmented structure. Also, torque of the brains, or any shape asymmetries, that could exist between studied groups, can affect the main direction of the bundle, threshold, and thus effect the anisotropy results.

As opposed to the ROI approach, where there is a driving clinical hypothesis prior to the measurements, a voxel based strategy is more exploratory and is suitable for identifying unpredicted/unhypothesized areas of abnormal white matter morphology. This method requires data to be spatially warped (or normalized) to the atlas template. Spatial normalization involves the registration of images and the generation of a stereotaxic atlas that represents the statistical distribution of the group at each voxel (Guimond et al., 2001; Friston et al., 1995; Mazziotta et al., 1995; Thompson et al., 1997). The registration of diffusion tensor images, is usually performed in a similar way to the registration of T1-weighted or SPGR MR images. That is, T2-weighted MR images are used to estimate deformation fields or transformation functions in order to minimize the intensity difference between the template and the normalized image. With the estimated transformation function, the morphology of the diffusion images is then deformed to fit a stereotaxic space. Since T2 structural images do not include information relevant to the orientation and direction of the fiber tracts, the registration of these types of images to the atlas template usually performs poorly, with significant residual miss-registration error due to differences in fiber tracts location, shape and size, as well as anatomical variability that can not be captured with information provided by structural images. For this reason, voxelbased quantification of diffusion tensor images requires more sophisticated spatial normalization that takes advantage of the directional information provided by the full tensor (Park et al., 2003).

\section{Applications of DTI to schizophrenia research}

As noted previously, based on geometry and the degree of anisotropy loss, white matter tract alterations, such as dislocation, disruption or disorganization can be documented. The crosssectional size of these pathways, as well as diffusion anisotropy measured within the bundle, yield a quantitative measure of connectivity between different brain regions. In fact, the tissue properties of white matter fiber tracts, including the density of the fibers, the fiber diameter, the thickness of the myelin sheaths, and the directionality (or coherence) of the fibers in each voxel, all affect the diffusion of water molecules, thereby providing useful information about white matter fiber tract abnormalities.

Of note, a number of studies, using a wide spectrum of methods, suggest that disrupted connectivity of widely distributed brain circuits is likely characteristic of schizophrenia (Volkow et al., 1988; Friston and Frith, 1995; McGuire and Frith, 1996; Weinberger et al., 1992; Fletcher et al., 1999). DTI thus has the potential to provide important new information about links between anatomical abnormalities and clinical symptoms observed in this disease. To date, 18 studies have examined white matter in schizophrenia using diffusion tensor imaging 
technique. Despite the consistency of the earliest DTI findings (first five DTI studies showed diffusion abnormalities in schizophrenia), five more recent studies do not show any statistical differences between schizophrenics and healthy controls. One reason for this lack of consistency in findings is likely due to the fact that there are no set standards for data acquisition and analysis and the methods of post-processing the data are also variable. In addition, small sample size, small ROI, lower S/N ratio due to smaller number of repetitions, acquisition angles, scanner gradient performance, greater partial volume effect due to the lower image resolution, as well as the use of anisotropic voxels, will skew the FA measurements, and decrease the statistical power of the DTI analysis. DTI findings in schizophrenia must, therefore, be understood within the context of the acquisition parameters used as well as in the context of the different methodological strategies adopted by different investigators. These differences are highlighted, below.

\section{DTI schizophrenia findings}

In order to compare diffusion anisotropy between control subjects and schizophrenics, most studies reported in the literature have used fractional anisotropy (FA). Only one study has used relative anisotropy (RA), (Buchsbaum et al., 1998) and only one study has used inter-voxel coherence as an index of diffusion (Kalus et al., 2004). In comparison with FA, RA maps show lower visual contrast between white and gray matter areas of the brain, since RA tends to be less sensitive to anisotropy changes in midrange values than the more popular FA index (Lim et al., 1999). In areas with higher diffusion anisotropy (fiber tracts), and lower diffusion anisotropy (gray matter), both indices perform similarly. Of note, an index of inter-voxel coherence for each voxel, as used by Kalus et al. (2004), takes into account not only diffusion properties within this particular voxel, but also in the adjacent voxels. This index tends to reflect better the fiber organization in very dense, small fiber bundles (see also Fig. 3). As an additional measure of fiber density and organization, mean diffusion along all directions, or mean diffusivity has also been estimated in several $(n=7)$ studies (see Table 1 ).

Decreased FA, along with increased diffusivity within prefrontal and temporal lobes, as well as abnormalities within the fiber bundles connecting these regions (including uncinate fasciculus, cingulum bundle and arcuate fasciculus) are the most frequent positive findings in schizophrenia studies (see Table 1). For example, uncinate fasciculus abnormalities were detected with small ROI defined by a directional threshold method (Kubicki et al., 2002a), and this finding has been confirmed by a voxel based morphometry analysis (Burns et al., 2003). Cingulum bundle abnormalities have also been reported in schizophrenia using both fixed size small ROI (Sun et al., 2003;Wang et al., 2004), and a directional threshold method (Kubicki et al., 2003). In addition, changes in the genu, but not the splenium of the corpus callosum, have been reported in schizophrenia using the fixed, small ROI method (Foong et al., 2000), and using VBM analysis (Agartz et al., 2001;Ardekani et al., 2003) although this finding was not confirmed in either another VBM analysis that used the same sample as the small ROI method (Foong et al., 2002), or in another study using small ROI and a larger sample size (Sun et al., 2003; see also Table 1). Additionally, internal capsule diffusion abnormalities have been detected in one VBM study (Buchsbaum et al., 1998), but not in a small ROI study (Sun et al., 2003) nor in two other VBM studies (Agartz et al., 2001;Foong et al., 2002). Further, two studies have investigated differences in the cerebellar peduncles in schizophrenia compared to controls (Wang et al., 2003;Okugawa et al., 2004), and one did not show differences, while the other demonstrated decrease of the FA in the middle cerebellar peduncles in schizophrenia subjects. In addition, two studies have reported widespread diffusion abnormalities in all tested regions (parietal, temporal, prefrontal and occipital white matter) when a fixed, large ROI method was used (Lim et al., 1999;Minami et al., 2003; see also Table 1). So far, only one study (Hubl et al., 2004), using VBM and relatively small number of subjects $(n=13)$, demonstrated increase of FA in schizophrenics with auditory hallucinations. 
Of particular note, all DTI studies of schizophrenia thus far, except one with negative findings in hippocampus that included 7 first episode schizophrenics (Begre et al., 2003), have examined chronic schizophrenia samples. Furthermore, medication was shown, to be related to left frontal white matter anisotropy (Minami et al., 2003), and in another study, to middle cerebellar peduncle (Okugawa et al., 2004). These two factors suggest that in subsequent studies there is a need to study more carefully the effects of medication, as well as to include first episode patients, who are either medication naïve or who have limited psychotropic exposure. In addition, the age of patients diagnosed with schizophrenia and the age of controls has not been observed thus far to correlate with DTI measures. Nonetheless, a recently published, wellcontrolled study, that included a large healthy population, has demonstrated a relationship between FA, diffusivity, and age (Pfefferbaum and Sullivan, 2003). This latter finding suggests that measures of diffusion may change with age and it will be useful to understand white matter changes across the age span in both normal controls and in pathological populations, as changes in aging that are evinced in normal controls may be quite different in pathological populations such as schizophrenia.

Some interesting correlations between diffusion measures and clinical and neuropsychological measures have also emerged in DTI studies of schizophrenia. Specifically, prefrontal white matter anisotropy has been reported to be correlated with negative symptoms, impulsiveness and aggressiveness (Hoptman et al., 2002; Wolkin et al., 2003). Additionally, FA for frontaltemporal connections have been reported to be correlated with measures of errors in executive functions related to performance monitoring (as measured by Wisconsin Card Sorting Test; WCST) - cingulate bundle, and declarative-episodic verbal memory (as measured by Wechsler Memory Scale-III; WMS-III) - uncinate fasciculus (Kubicki et al., 2002a, 2003). While exploratory, and not studied frequently in DTI studies (VBM methods so far have not explored clinical correlations at all), these findings demonstrate the sensitivity of the diffusion measurements to white matter abnormalities, and further provide evidence of the potential role that DTI-neuropsychological correlates can play in understanding disease-related cognitive disturbance. Moreover, the rather strong dissociation of UF-WMS-III and CB-WCST underscores the value of DTI in delineating important and distinct brain networks in patients with schizophrenia.

\section{Implications, conclusions and future directions}

Preliminary DTI results, data from other MR techniques, such as magnetization transfer techniques and MR relaxation measurements (both sensitive to the myelin abnormalities), as well as MR spectroscopy (measuring neuronal markers), in combination with pathomorphological data, all suggest that white matter pathways play an important role in the neuropathology of schizophrenia, and are likely related to the myriad of clinical symptoms observed in this disorder. DTI findings to date are interesting, but methodological limitations, discussed here as well as elsewhere, limit our ability to conclude, with certainty, the nature of white matter pathology in schizophrenia. What we do know is that DTI provides a new window of opportunity to evaluate white matter in a manner not possible with conventional MRI. However, we do not as yet know whether the abnormalities observed to date reflect a decrease in number of axons, decreased axonal diameter, thinner myelination sheaths, less coherent fibers, more fiber crossings, or simply more noise in the DTI data.

Despite these limitations, and the many questions that remain with respect to how best to understand the diffusion findings in schizophrenia, we believe that DTI shows much promise for illuminating our understanding of how specific brain regions are connected and how such connectivity may be relevant to our understanding of functional abnormalities, both cognitive, and clinical, observed in schizophrenia. We further believe that future research, which uses high magnet fields, and fast, low distortion techniques that produce high resolution and high 
SNR diffusion images, will lead to a further understanding of white matter pathology in schizophrenia. Other advances that we will likely have over the next few years are improved fiber tractography methods that are optimized and validated, and then applied to these high resolution data. Such studies will likely benefit from the acquisition of contiguous, isotropic voxels (so far most of the clinical studies use anisotropic voxels with gaps between slices, which biases the major diffusion of the tensor and affects the contiguity of the fiber tract thus skewing the fiber tract direction), and diffusion images sampled in multiple directions. With reliable fiber tracking, it should be relatively easy to define brain regions of interest along the fiber bundles, and to measure both mean diffusion anisotropy, as well as cross-sectional area of these fiber bundles.

Fig. 6 provides an example of work that will likely assist us in evaluating fiber bundles in patients with schizophrenia. Here, automated fiber tracking procedure (described in detail in Park et al., 2003) has been used to create major white matter fiber tracts. In brief, all white matter voxels have been used as seeding points, and then fiber bundles were created by following the largest eigenvectors of the diffusion tensor using the Runge-Kutta algorithm (Basser et al., 2000). Next, FA values for each voxel within the bundles have been calculated, and voxels have been color coded based on their FA values. This work will need to be validated in postmortem tissue so that we will have a clearer understanding of the pathological processes detectable using DTI. Of further note, with today's scanning techniques it is clear that results from fiber tractography do not describe the spatial extent of individual axons but instead describe the average diffusion properties in white tissue at the scale of a millimeter. Thus, the diffusion tensors at this scale should be regarded as estimators of local diffusion properties and tractography as a visualization of features in this field. Validation of the features captured in the tensor field is thus very challenging since the definition of the tissue properties corresponding to the diffusion field is not well understood. However, the similarities between DT-MRI tractography and real white matter fiber architecture in the human brain are compelling even at today's limited resolution.

Future studies will also need to include an evaluation of first episode psychotic patients, so as to provide a sample of patients who have limited exposure to psychotropic medications. Additionally, samples need to be comprised of both males and females, as there is clear evidence to suggest that the timing, course, and clinical features of schizophrenia are manifested quite differently in males and females, and this may be related to brain differences (Goldstein et al., 2002). It will also be important to evaluate a representative sample of normal controls across age, as white matter changes with age, and such changes may be quite different in pathological populations such as schizophrenia. Future DTI studies need also to include other imaging techniques, which highlight white matter pathologies, such as proton spectroscopy, magnetization transfer techniques and relaxation times measurements.

Moreover, such studies should be conducted in concert with functional MRI and PET imaging in order to characterize and to understand more fully both functional and structural abnormalities in schizophrenia. Voxel based analyses will likely still be performed in future studies, as this methodological approach remains the simplest way to define possible regions of pathology, although it should be accompanied by ROI analyses, as well as utilize better, more precise registration strategies.

Finally, we note that DTI was introduced to clinical imaging only in 1995, and that such a new imaging tool has only just begun to be explored with respect to what it has to offer in documenting white matter abnormalities in schizophrenia. We are thus only at the beginning stages of what will likely be further technological advances, as well as new white matter findings in schizophrenia. 


\section{Acknowledgments}

The authors thank Marie Fairbanks for her administrative assistance. They also gratefully acknowledge the support of the National Alliance for Research on Schizophrenia and Depression (MK), the National Institute of Health (R03 MH068464-01 to MK, K02 MH 01110 and R01 MH 50747 to MES, R01 NS 39335 to SEM and R01 MH 40799 to RWM), the Post-doctoral Fellowship Program of Korea Science and Engineering Foundation (KOSEF) (HJP), the Department of Veterans Affairs Merit Awards (MES, RWM), and the National Center for Research Resources (NIH P41 RR13218 to RK).

\section{References}

Agartz I, Andersson JL, Skare S. Abnormal brain white matter in schizophrenia: a diffusion tensor imaging study. Neuroreport 2001;12(10):2251-4. [PubMed: 11447344]

Akbarian S, Kim JJ, Potkin SG, Hetrick WP, Bunney WE Jr, Jones EG. Maldistribution of interstitial neurons in prefrontal white matter of the brains of schizophrenic patients. Archives of General Psychiatry 1996;53(5):425-36. [PubMed: 8624186]

Ardekani BA, Nierenberg J, Hoptman MJ, Javitt DC, Lim KO. MRI study of white matter diffusion anisotropy in schizophrenia. Neuroreport 2003;14(16):2025-9. [PubMed: 14600491]

Bammer R, Auer M, Keeling SL, Augustin M, Stables LA, Prokesch RW, et al. Diffusion tensor imaging using single-shot SENSE-EPI. Magnetic Resonance in Medicine 2002;48(1):128-36. [PubMed: 12111940]

Basser PJ. Inferring microstructural features and the physiological state of tissues from diffusionweighted images. NMR in Biomedicine 1995;8(7-8):333-44. [PubMed: 8739270]

Basser PJ, Mattiello J, LeBihan D. MR diffusion tensor spectroscopy and imaging. Biophysical Journal 1994;66(1):259-67. [PubMed: 8130344]

Basser PJ, Pajevic S, Pierpaoli C, Duda J, Aldroubi A. In vivo fiber tractography using DT-MRI data. Magnetic Resonance in Medicine 2000;44(4):625-32. [PubMed: 11025519]

Beaulieu C, Allen P. Determinants of anisotropic water diffusion in nerves. MRM 1994;31:394-400.

Begre S, Federspiel A, Kiefer C, Schroth G, Dierks T, Strik WK. Reduced hippocampal anisotropy related to anteriorization of alpha EEG in schizophrenia. Neuroreport 2003;14(5):739-42. [PubMed: 12692474]

Benes, FM. Relationship of cingulate cortex to schizophrenia and other psychiatric disorders.. In: Vogt, BA.; Gabriel, M., editors. Neurobiology of cingulate cortex and limbic thalamus: a comprehensive handbook. Birkhauser; Boston: 1993. p. 581-605.

Breier A, Buchanan RW, Elkashef A, Munson RC, Kirkpatrick B, Gellad F. Brain morphology and schizophrenia. A magnetic resonance imaging study of limbic, prefrontal cortex, and caudate structures. Archives of General Psychiatry 1992;49(12):921-6. [PubMed: 1449382][see comments]

Buchsbaum MS, Tang CY, Peled S, Gudbjartsson H, Lu D, Hazlett EA, et al. MRI white matter diffusion anisotropy and PET metabolic rate in schizophrenia. Neuroreport 1998;9(3):425-30. [PubMed: 9512384]

Burns J, Job D, Bastin ME, Whalley H, Macgillivray T, Johnstone EC, et al. Structural disconnectivity in schizophrenia: a diffusion tensor magnetic resonance imaging study. British Journal of Psychiatry 2003;182:439-43. [PubMed: 12724248]

Davis KL, Stewart DG, Friedman JI, Buchsbaum M, Harvey PD, Hof PR, et al. White matter changes in schizophrenia: evidence for myelin-related dysfunction. Archives of General Psychiatry 2003;60(5): 443-56. [PubMed: 12742865]

Fletcher P, McKenna PJ, Friston KJ, Frith CD, Dolan RJ. Abnormal cingulate modulation of frontotemporal connectivity in schizophrenia. Neuroimage 1999;9(3):337-42. [PubMed: 10075903]

Foong J, Maier M, Clark CA, Barker GJ, Miller DH, Ron MA. Neuropathological abnormalities of the corpus callosum in schizophrenia: a diffusion tensor imaging study. Journal of Neurology Neurosurgery and Psychiatry 2000;68(2):242-4.

Foong J, Symms MR, Barker GJ, Maier M, Miller DH, Ron MA. Investigating regional white matter in schizophrenia using diffusion tensor imaging. Neuroreport 2002;13(3):333-6. [PubMed: 11930133]

Frank LR. Characterization of anisotropy in high angular resolution diffusion-weighted MRI. Magnetic Resonance in Medicine 2002;47(6):1083-99. [PubMed: 12111955] 
Friston K, Holmes A, Worsley KJ, Poline JP, Frith CD, Frackowiak RSJ. Statistical parametric maps in functional imaging: a general linear approach. Human Brain Mapping 1995;2:189-210.

Friston KJ, Frith CD. Schizophrenia: a disconnection syndrome? Clinical Neuroscience 1995;3(2):8997. [PubMed: 7583624]

Goldstein JM, Seidman LJ, OÕBrien LM, Horton NJ, Kennedy DN, Makris N, et al. Impact of normal sexual dimorphisms on sex differences in structural brain abnormalities in schizophrenia assessed by magnetic resonance imaging. Archives of General Psychiatry 2002;59(2):154-64. [PubMed: 11825137]

Gudbartsson H, Maier S, Mulkern R. Line scan diffusion imaging. Magnetic Resonance in Medicine 1996;36:509-19. [PubMed: 8892201]

Guimond A, Roche A, Ayache N, Meunier J. Three-dimensional multimodal brain warping using the demons algorithm and adaptive intensity corrections. IEEE Transactions in Medical Imaging 2001;20 (1):58-69.

Hagmann P, Reese TG, Tseng W-YI, Meuli R, Thiran J-P, Wedeen VJ. Diffusion spectrum imaging tractography in complex cerebral white matter: an investigation of the centrum cemiovale. Proceedings of the International Society of Magnetic Resonance in Medicine 2004;11:623.

Hakak Y, Walker JR, Li C, Wong WH, Davis KL, Buxbaum JD, et al. Genome-wide expression analysis reveals dysregulation of myelination-related genes in chronic schizophrenia. Proceedings of the National Academy of Sciences of United States America 2001;98(8):4746-51.

Highley JR, Esiri MM, McDonald B, Cortina-Borja M, Herron BM, Crow TJ. The size and fibre composition of the corpus callosum with respect to gender and schizophrenia: a post-mortem study. Brain 1999a;122(Pt 1):99-110. [PubMed: 10050898]

Highley JR, Esiri MM, McDonald B, Roberts HC, Walker MA, Crow TJ. The size and fiber composition of the anterior commissure with respect to gender and schizophrenia. Biological Psychiatry 1999b; 45(9):1120-7. [PubMed: 10331103]

Highley JR, Walker MA, Esiri MM, Crow TJ, Harrison PJ. Asymmetry of the uncinate fasciculus: a postmortem study of normal subjects and patients with schizophrenia. Cerebral Cortex 2002;12(11): 1218-24. [PubMed: 12379610]

Hof PR, Haroutunian V, Friedrich VL Jr, Byne W, Buitron C, Perl DP, et al. Loss and altered spatial distribution of oligodendrocytes in the superior frontal gyrus in schizophrenia. Biological Psychiatry 2003;53:1075-85. [PubMed: 12814859]

Hoptman MJ, Volavka J, Johnson G, Weiss E, Bilder RM, Lim KO. Frontal white matter microstructure, aggression, and impulsivity in men with schizophrenia: a preliminary study. Biological Psychiatry 2002;52(1):9-14. [PubMed: 12079725]

Hubl D, Koenig T, Strik W, Federspiel A, Kreis R, Boesch C, et al. Pathways that make voices: white matter changes in auditory hallucinations. Archives of General Psychiatry 2004;61:658-68. [PubMed: 15237078]

Huppi PS, Maier SE, Peled S, Zientara GP, Barnes PD, Jolesz FA, et al. Microstructural development of human newborn cerebral white matter assessed in vivo by diffusion tensor magnetic resonance imaging. Pediatric Research 1998;44(4):584-90. [PubMed: 9773850]

Kalus P, Buri C, Slotboom J, Gralla J, Remonda L, Dierks T, et al. Volumetry and diffusion tensor imaging of hippocampal subregions in schizophrenia. Neuroreport 2004;15(5):867-71. [PubMed: 15073533]

Kraepelin, E. Dementia praecox. Barclay, E., editor. Churchill Livingstone Inc.; New York: 19191971. SBTrans.

Kubicki M, Maier SE, Westin CF, Mamata H, Ersner-Hershfield H, Estepar R, et al. Comparison of single-shot echo-planar and line scan protocols for diffusion tensor imaging. Academic Radiology 2004;11(2):224-32. [PubMed: 14974598]

Kubicki M, Westin CF, Maier SE, Frumin M, Nestor PG, Salisbury DF, et al. Uncinate fasciculus findings in schizophrenia: a magnetic resonance diffusion tensor imaging study. American Journal of Psychiatry 2002a;159(5):813-20. [PubMed: 11986136]

Kubicki M, Westin CF, Maier SE, Mamata H, Frumin M, Ersner-Hershfield H, et al. Diffusion tensor imaging and its application to neuropsychiatric disorders. Harvard Review of Psychiatry 2002b;10 (6):324-36. [PubMed: 12485979] 
Kubicki M, Westin CF, Nestor PG, Wible CG, Frumin M, Maier SE, et al. Cingulate fasciculus integrity disruption in schizophrenia: a magnetic resonance diffusion tensor imaging study. Biological Psychiatry 2003;54(11):1171-80. [PubMed: 14643084]

Kumra S, Ashtari M, McMeniman M, Vogel J, Augustin R, Becker DE, et al. Reduced frontal white matter integrity in early-onset schizophrenia: a preliminary study. Biological Psychiatry 2004;55:1138-45. [PubMed: 15184032]

Le Bihan D, Breton E, Lallemand D, Grenier P, Cabanis E, Laval-Jeantet M. MR imaging of intravoxel incoherent motions: application to diffusion and perfusion in neurologic disorders. Radiology 1986;161(2):401-7. [PubMed: 3763909]

Lim KO, Hedehus M, Moseley M, de Crespigny A, Sullivan EV, Pfefferbaum A. Compromised white matter tract integrity in schizophrenia inferred from diffusion tensor imaging. Archives of General Psychiatry 1999;56(4):367-74. [PubMed: 10197834]

Maier SE. Slab scan diffusion imaging. Magnetic Resonance in Medicine 2001;46(6):1136-43. [PubMed: 11746580]

Makris N, Worth AJ, Sorensen AG, Papadimitriou GM, Wu O, Reese TG, et al. Morphometry of in vivo human white matter association pathways with diffusion-weighted magnetic resonance imaging. Annals of Neurology 1997;42:951-62. [PubMed: 9403488]

Mazziotta JC, Toga AW, Evans A, Fox P, Lancaster J. A probabilistic atlas of the human brain: theory and rationale for its development. The International Consortium for Brain Mapping (ICBM). Neuroimage 1995;2(2):89-101. [PubMed: 9343592]

McGuire PK, Frith CD. Disordered functional connectivity in schizophrenia [editorial]. Psychological Medicine 1996;26(4):663-7. [PubMed: 8817700]

Minami T, Nobuhara K, Okugawa G, Takase K, Yoshida T, Sawada S, et al. Diffusion tensor magnetic resonance imaging of disruption of regional white matter in schizophrenia. Neuropsychobiology 2003;47(3):141-5. [PubMed: 12759557]

Ordidge RJ, Helpern JA, Qing ZX, Knight RA, Nagesh V. Correction of motional artifacts in diffusionweighted MR images using navigator echoes. Magnetic Resonance in Medicine 1994;12(3):455-60.

Okugawa G, Nobuhara K, Minami T, Tamagaki C, Takase K, Sugimoto T, et al. Subtle disruption of the middle cerebellar peduncles in patients with schizophrenia. Neuropsychobiology 2004;50:119-23. [PubMed: 15292664]

Paillere-Martinot M, Caclin A, Artiges E, Poline JB, Joliot M, Mallet L, et al. Cerebral gray and white matter reductions and clinical correlates in patients with early onset schizophrenia. Schizophrenia Research 2001;50(1-2):19-26. [PubMed: 11378311]

Park HJ, Kubicki M, Shenton ME, Guimond A, McCarley RW, Maier SE, et al. Spatial normalization of diffusion tensor MRI using multiple channels. Neuroimage 2003;20(4):1995-2009. [PubMed: 14683705]

Peled S, Gudbjartsson H, Westin CF, Kikinis R, Jolesz FA. Magnetic resonance imaging shows orientation and asymmetry of white matter fiber tracts. Brain Research 1998;780:27-33. [PubMed: 9473573]

Pfefferbaum A, Sullivan EV. Increased brain white matter diffusivity in normal adult aging: relationship to anisotropy and partial voluming. Magnetic Resonance in Medicine 2003;49(5):953-61. [PubMed: 12704779]

Pfefferbaum A, Sullivan EV, Hedehus M, Adalsteinsson E, Lim KO, Moseley M. In vivo detection and functional correlates of white matter microstructural disruption in chronic alcoholism. AlcoholismClinical and Experimental Research 2000;24(8):1214-21.

Pierpaoli C, Jezzard P, Basser PJ, Barnett A, Di Chiro G. Diffusion tensor MR imaging of the human brain. Radiology 1996;201:637-48. [PubMed: 8939209]

Reese TG, Weisskoff RM, Smith RN, Rosen BR, Dinsmore RE, Wedeen VJ. Imaging myocardial fiber architecture in vivo with magnetic resonance. Magnetic Resonance in Medicine 1995;34:786-91. [PubMed: 8598805]

Shenton ME, Dickey CC, Frumin M, McCarley RW. A review of MRI findings in schizophrenia. Schizophrenia Research 2001;49(1-2):1-52. [PubMed: 11343862]

Sigmudsson T, Suckling J, Maier M, Williams SCR, Bullmore ET, Greenwood KE, et al. Structural abnormalities in frontal, temporal, and limbic regions and interconnecting white matter tracts in 
schizophrenic patients with prominent negative symptoms. American Journal of Psychiatry 2001;158:234-43. [PubMed: 11156806]

Steel RM, Bastin ME, McConnell S, Marshall I, Cunningham-Owens DG, Lawrie SM, Johnstone EC, Best JJK. Diffusion tensor imaging (DTI) and proton magnetic resonance spectroscopy (H MRS) in schizophrenic subjects and normal controls. Psychiatry Research: Neuroimaging Section 2001;106:161-70.

Stejskal EO, Tanner JE. Spin diffusion measurements: spin echoes in the presence of a time-dependent field gradient. Journal of Chemical Physics 1965;42:288-92.

Suddath RL, Christison GW, Torrey EF, Casanova MF, Weinberger DR. Anatomical abnormalities in the brains of monozygotic twins discordant for schizophrenia. New England Journal of Medicine 1990;322(12):789-94. [PubMed: 2308615]

Sun Z, Wang F, Cui L, Breeze J, Du X, Wang X, et al. Abnormal anterior cingulum in patients with schizophrenia: a diffusion tensor imaging study. Neuroreport 2003;14(14):1833-6. [PubMed: 14534430]

Thompson PM, MacDonald D, Mega MS, Holmes CJ, Evans AC, Toga AW. Detection and mapping of abnormal brain structure with a probabilistic atlas of cortical surfaces. Journal of Computer Assisted Tomography 1997;21(4):567-81. [PubMed: 9216760]

Trouard TP, Theilmann RJ, Altbach MI, Gmitro AF. High-resolution diffusion imaging with DIFRADFSE (diffusion-weighted radial acquisition of data with fast spin-echo) MRI. Magnetic Resonance in Medicine 1999;42(1):11-8. [PubMed: 10398944]

Tuch DS, Reese TG, Wiegell MR, Makris N, Belliveau JW, Wedeen VJ. High angular resolution diffusion imaging reveals intravoxel white matter fiber heterogeneity. Magnetic Resonance in Medicine 2002;48(4):577-82. [PubMed: 12353272]

Turner R, Le Bihan D, Maier J, Vavrek R, Hedges LK, Pekar J. Echo-planar imaging of intravoxel incoherent motion. Radiology 1990;177(2):407-14. [PubMed: 2217777]

Uranova N, Orlovskaya D, Vikhreva O, Zimina I, Kolomeets N, Vostrikov V, et al. Electron microscopy of oligodendroglia in severe mental illness. Brain Research Bulletin 2001;55(5):597-610. [PubMed: 11576756]

Uranova NA, Vostrikov VM, Orlovskaya DD, Rachmanova VI. Oligodendroglial density in the prefrontal cortex in schizophrenia and mood disorders: a study from the Stanley Neuropathology Consortium. Schizophrenia Research 2004;67(2-3):269-75. [PubMed: 14984887]

Volkow ND, Wolf AP, Brodie JD, Cancro R, Overall JE, et al. Brain interactions in chronic schizophrenics under resting and activation conditions. Schizophrenia Research 1988;1(1):47-53. [PubMed: 3154506]

Wang F, Sun Z, Cui L, Du X, Wang X, Zhang H, et al. Anterior cingulum abnormalities in male patients with schizophrenia determined through diffusion tensor imaging. American Journal of Psychiatry 2004;161(3):573-5. [PubMed: 14992988]

Wang F, Sun Z, Du X, Wang X, Cong Z, Zhang H, et al. A diffusion tensor imaging study of middle and superior cerebellar peduncle in male patients with schizophrenia. Neuroscience Letters 2003;348(3): 135-8. [PubMed: 12932812]

Weinberger DR, Berman KF, Suddath R, Torrey EF. Evidence of dysfunction of a prefrontal-limbic network in schizophrenia: a magnetic resonance imaging and regional cerebral blood flow study of discordant monozygotic twins. American Journal of Psychiatry 149(7):890-7.

Wernicke, C. Grundrisse der Psychiatrie. Thieme; Leipzig: 1906.

Westin CF, Maier SE, Mamata H, Nabavi A, Jolesz FA, Kikinis R. Processing and visualization for diffusion tensor MRI. Medical Image Analysis 2002;6(2):93-108. [PubMed: 12044998]

Wible CG, Shenton ME, Hokama H, Kikinis R, Jolesz FA, Metcalf D, et al. Prefrontal cortex and schizophrenia. A quantitative magnetic resonance imaging study. Archives of General Psychiatry 1995;52(4):279-88. [PubMed: 7702444]

Wolkin A, Choi SJ, Szilagyi S, Sanfilipo M, Rotrosen JP, Lim KO. Inferior frontal white matter anisotropy and negative symptoms of schizophrenia: a diffusion tensor imaging study. American Journal of Psychiatry 2003;160:572-4. [PubMed: 12611842] 


\section{Anisotropic \\ diffusion $\left(\lambda_{1}>\lambda_{2}\right.$ and $\left.\lambda_{3}\right)$}

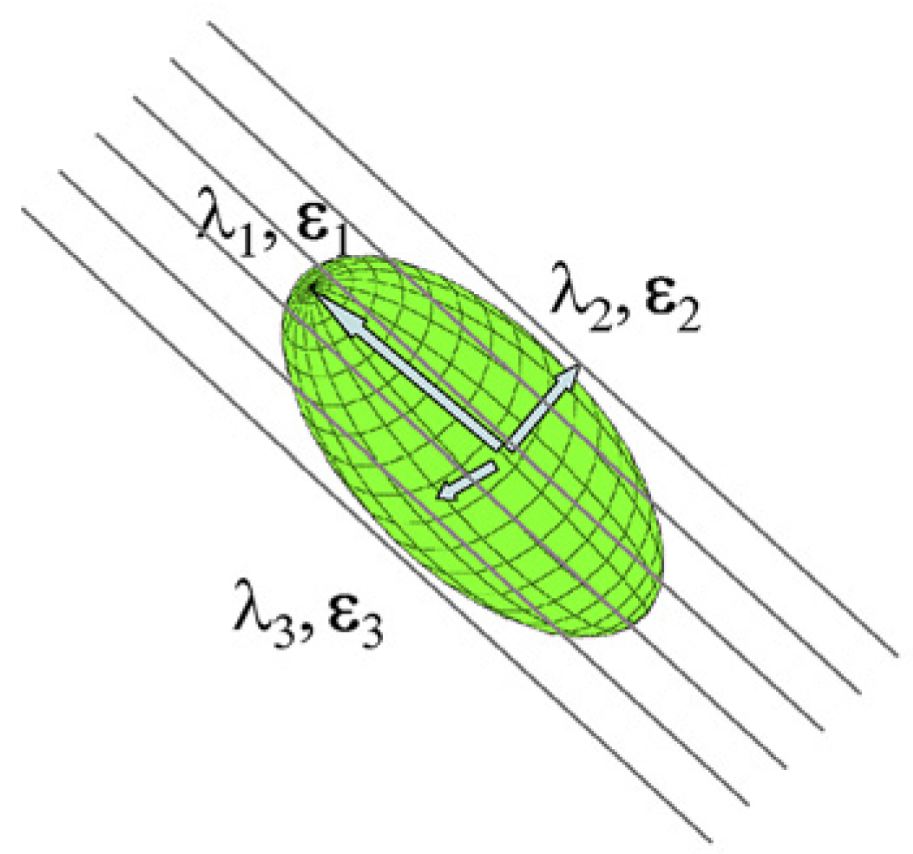

$$
\begin{aligned}
& \lambda_{1}, \lambda_{2}, \lambda_{3} \text { - eigenvalues of } \mathbf{D} \\
& \varepsilon_{1}, \varepsilon_{2}, \varepsilon_{3} \text { - eigenvectors of } \mathbf{D}
\end{aligned}
$$

Fig. 1.

Schematic of diffusion tensor imaging. 


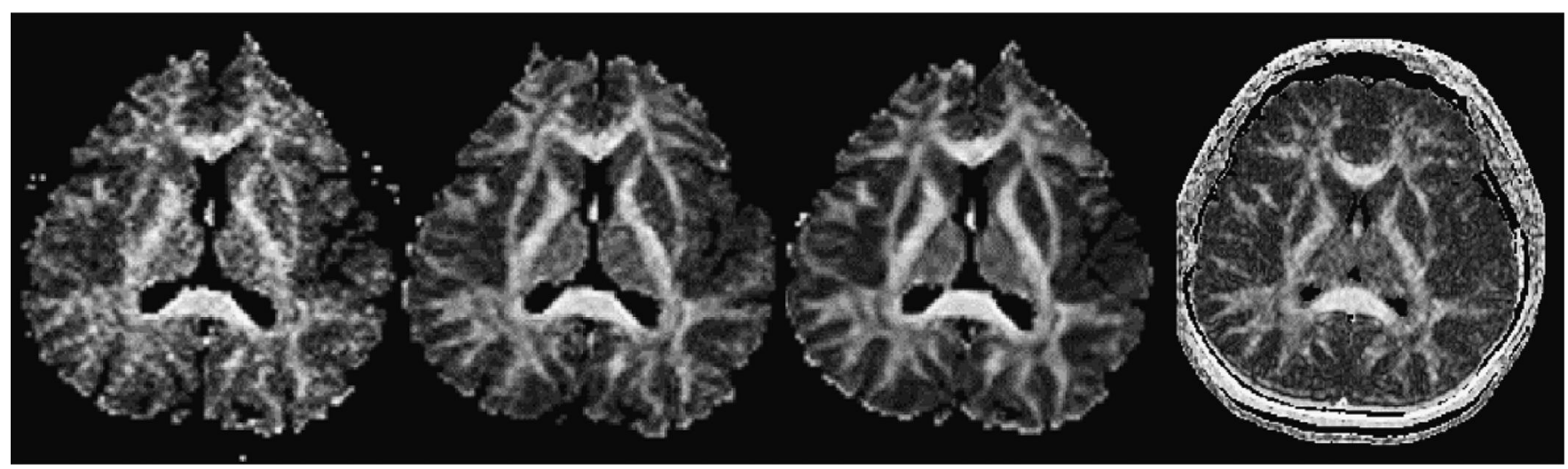

Fig. 2.

Influence of the number of excitations (NEX) on the data noise. 1, 4 and 16 acquisitions of single-shot EPI, and 1 acquisition of LSDI. Slice thickness $4 \mathrm{~mm}$, axial orientation. 

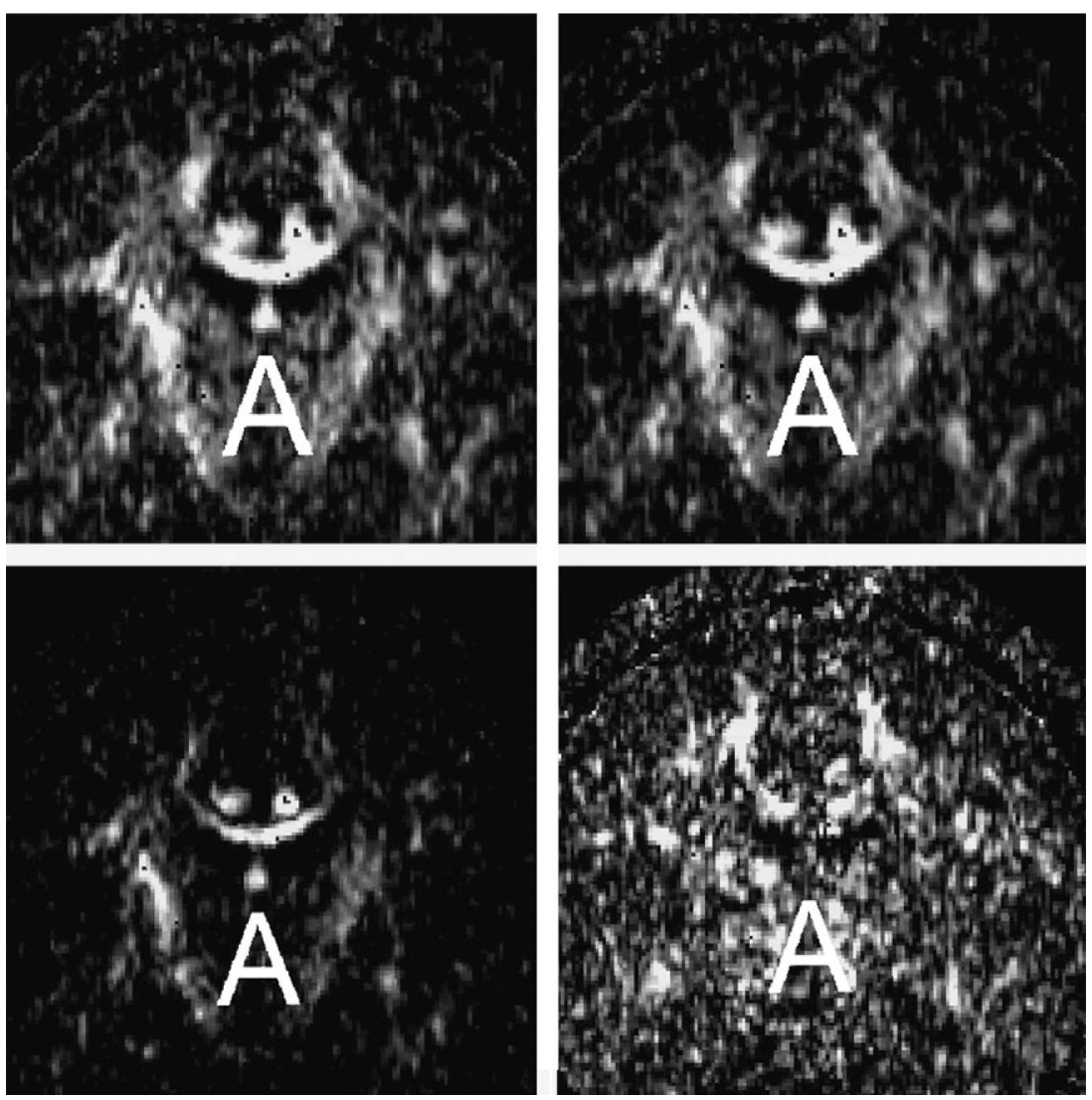

Fig. 3.

Different indices used to represent diffusion. Upper left - FA (the measure of the fraction of the magnitude of the tensor that can be ascribed to the anisotropic diffusion). Upper right - RA (normalized standard deviation representing the ratio of the anisotropic part of the tensor to its isotropic part). Lower left - TR (the sum of the eigenvalues of the tensor, or the sum of diffusion in all three principal directions). Lower right - linear measure of diffusion (representing the coherence of the fibers for each voxel) and spherical measure of diffusion (visualizing fiber crossings). 

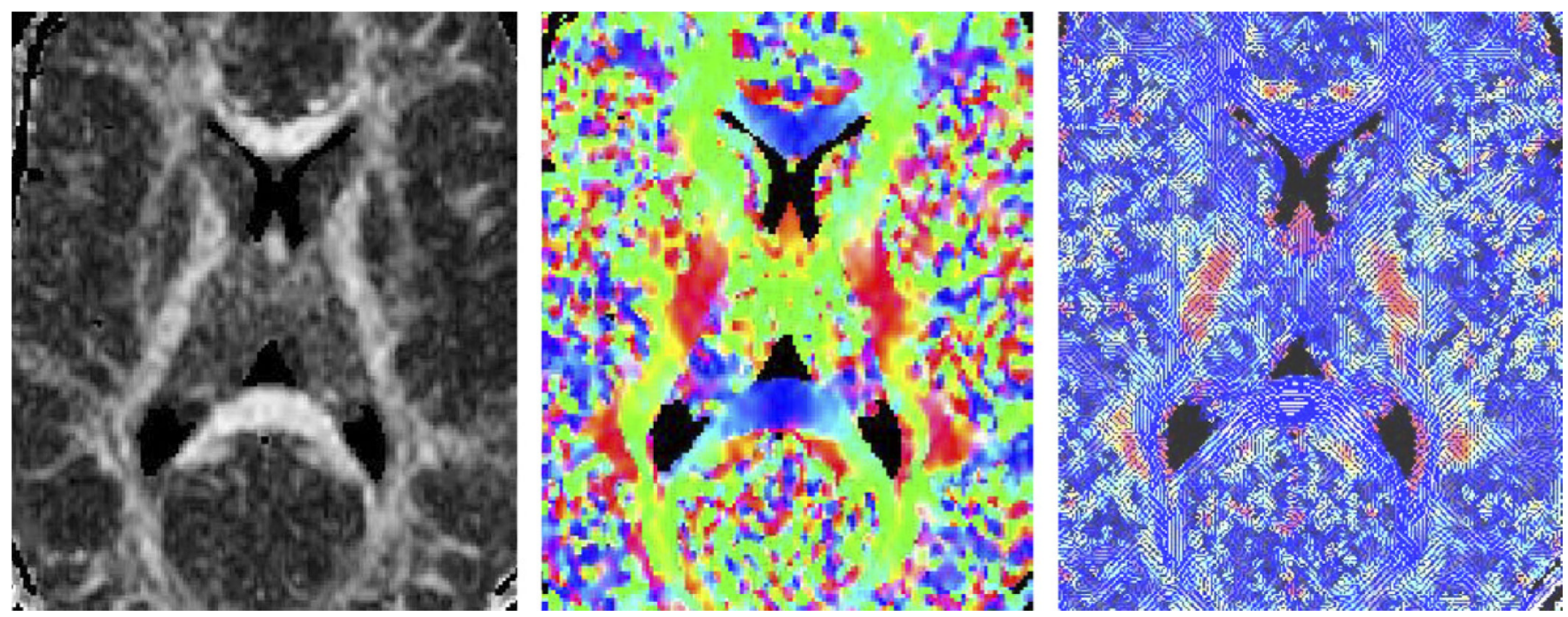

Fig. 4.

Different methods for visualizing diffusion tensor information - left panel - map of fractional anisotropy with higher intensity representing more anisotropic diffusion, middle panel - color map, where different intensities of the three colors indicate the size and the ADC in each of the three Cartesian directions; right panel - blue lines representing the in-plane component of the principal diffusion direction, and a color-coded out-of-plane component (more intuitive approach, where each line represents the main diffusion direction for local neighborhood, rather than for each voxel). 

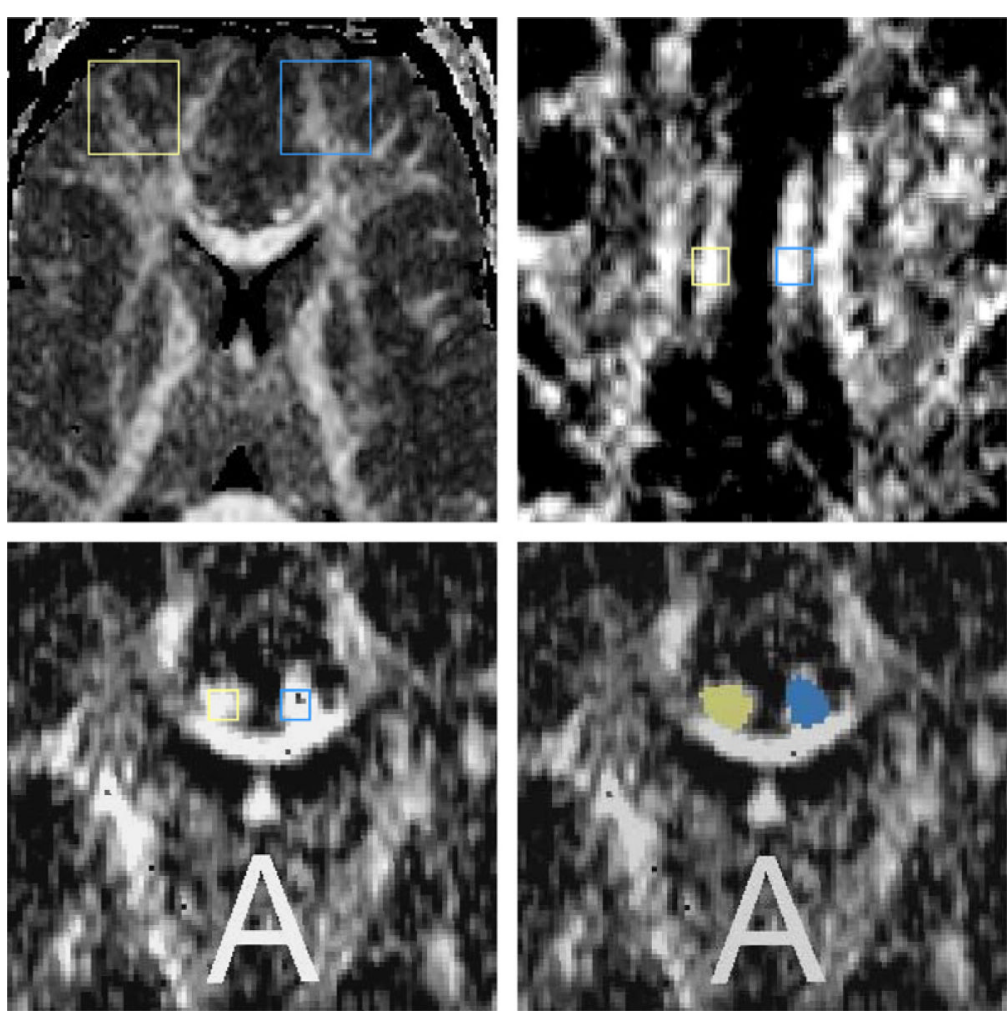

Fig. 5.

ROI methods for quantitative analysis of diffusion - upper-left-hand-side panel - Large ROI placed on the axial slice within the frontal lobe, upper right-hand-side panel - small fixed ROI placed on the axial slice within the cingulum bundle, lower left-hand-side panel - small, fixed ROI placed within the cingulum bundle on the coronal plane, and lower right-hand-side - small ROI of the cingulum bundle on the coronal slice based on the directional information. Yellow - right side, blue - left side. 


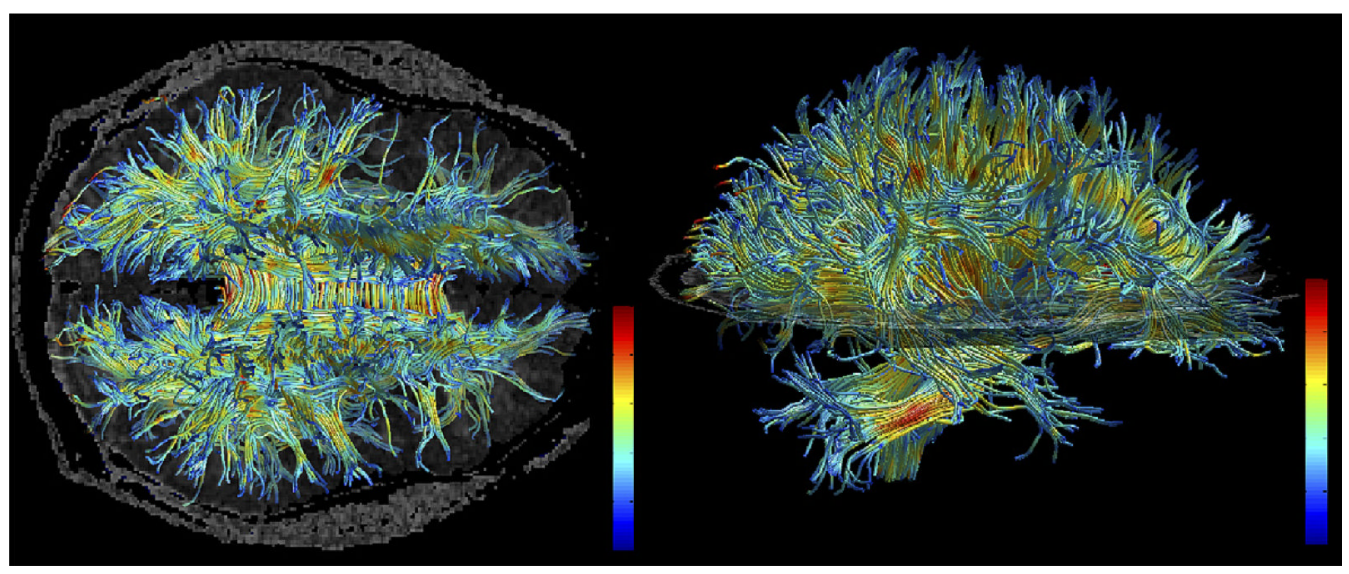

Fig. 6.

Example of fiber tracking. High resolution data acquired on 3 Tesla magnet and post-processed using automated tracking procedure. Voxels within fiber bundles are color coded according to their FA values (i.e., blue, low anisotropy; and red, high anisotropy). 


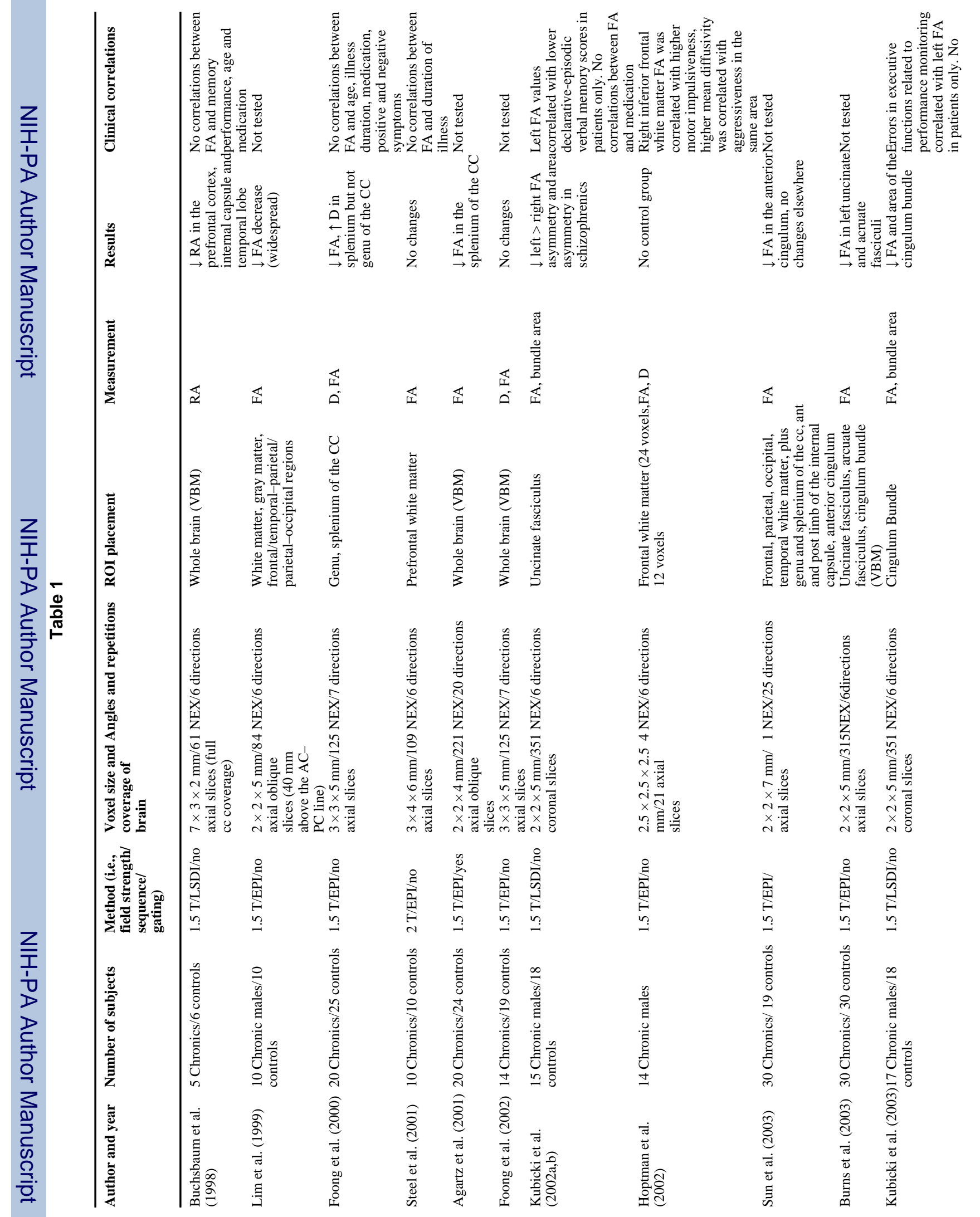




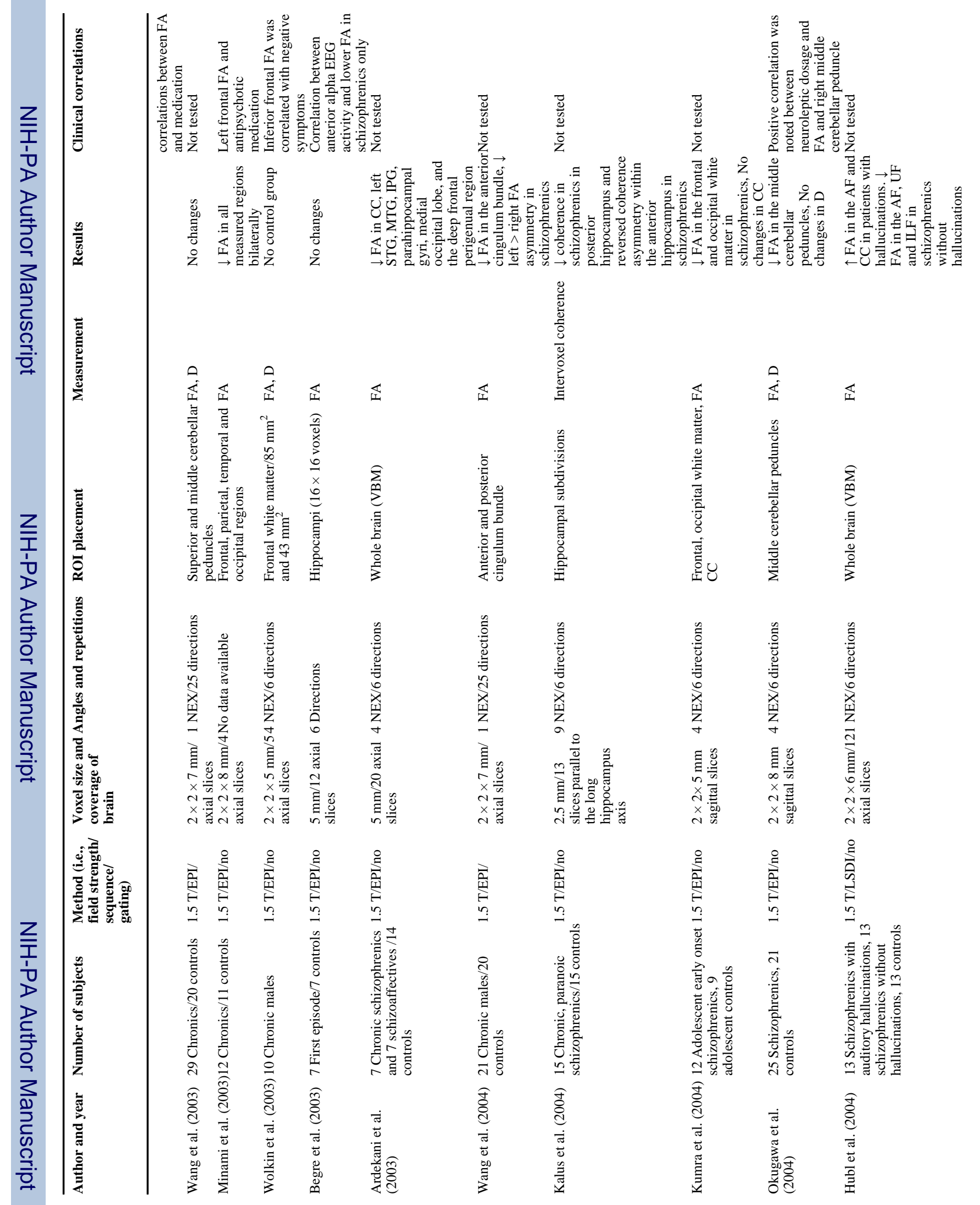




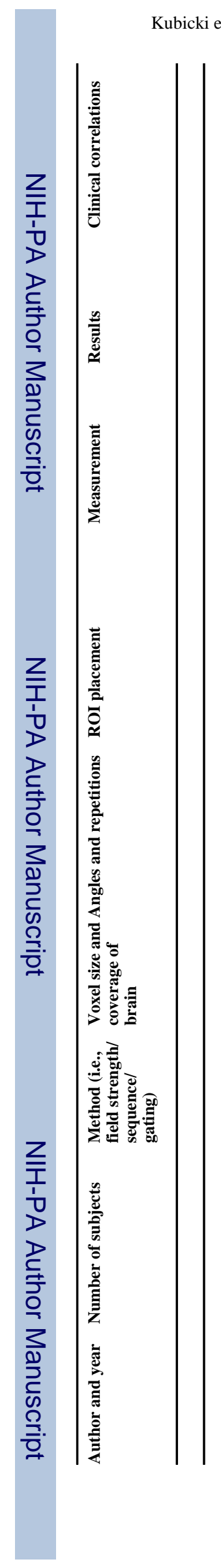

Page 23 